\title{
TV/Series
}

$3 \mid 2013$

Écho et reprise dans les séries télévisées (I) : Reprise et intermédialité

\section{Les constellations narratives. Que font les téléspectateurs des adaptations multimédiatiques des séries télévisées?}

\section{Sarah Sepulchre}

\section{QpenEdition}

\section{Journals}

Édition électronique

URL : http://journals.openedition.org/tvseries/729

DOI : $10.4000 /$ tvseries.729

ISSN : 2266-0909

Éditeur

GRIC - Groupe de recherche Identités et Cultures

Référence électronique

Sarah Sepulchre, "Les constellations narratives. Que font les téléspectateurs des adaptations multimédiatiques des séries télévisées? », TV/Series [En ligne], 3 | 2013, mis en ligne le 15 septembre 2013, consulté le 19 avril 2019. URL : http://journals.openedition.org/tvseries/729 ; DOI : 10.4000/ tvseries.729

\section{c) (1) $(9)$}

TV/Series est mis à disposition selon les termes de la licence Creative Commons Attribution - Pas d'Utilisation Commerciale - Pas de Modification 4.0 International. 


\title{
Les constellations narratives. Que font les téléspectateurs
} des adaptations multimédiatiques des séries télévisées?

\begin{abstract}
Les séries télévisées débordent de plus en plus du petit écran pour investir d'autres supports comme le livre, Internet, le cinéma, les jeux. Nous appelons "constellations" ces ensembles d'éléments qui semblent participer d'une même histoire. La première partie de l'article montre que cette notion est proche de celles de "transmédia" (Henry Jenkins). Ce qui semble au cœur de ces systèmes narratifs est la création de mondes diégétiques immersifs (Anne Besson). Le second volet de la contribution développe donc les notions d’immersion (Renée Bourassa, Jean-Marie Schaeffer) et de figure (Bertrand Gervais), qui nous permettront de conceptualiser les pratiques des lecteurs. Une étude de réception exploratoire basée sur des entretiens semi-directifs avec des amateurs de séries télévisées conclut ce texte. Elle montre que les consommateurs prennent en effet plaisir à prolonger la jouissance du monde diégétique par une immersion plus fictionnelle que technique. En revanche, les éléments qui alimentent l'univers ne sont pas toujours de nature fictionnelle car les lecteurs utilisent parfois des informations sur les acteurs ou sur les lieux du récit pour nourrir leur activité imaginative. Les constellations restent cependant des récits transmédiatiques faibles car les différentes entrées sont moins autonomes que dans les cas idéaux décrits par Henry Jenkins. Ces ensembles narratifs fonctionnent en effet davantage sur la base du modèle satellitaire présenté par Richard Saint-Gelais. Enfin, il apparait que la consommation de constellation est une véritable appropriation de l'univers fictionnel par les lecteurs.
\end{abstract}

$\mathrm{U}$ ne série peut être déclinée sur plusieurs médias : à la télévision, au cinéma, dans des comics, sur Internet, sur des sites Internet fictionnels, des profils Facebook ou des comptes Twitter de personnages, dans des jeux, des jeux vidéos, des romans, des romans écrits par des personnages, etc. Cougar Town (ABC, 2009-) est prolongée par des webisodes ${ }^{1}$ centrés sur les rêves d'Andy; Patty Hewes, le personnage principal de Damages (FX \& Audience Network, 2007-2012), écrit des tweets; Hank Schrader (Breaking Bad, AMC, 2008-2013) tient un blog; les romans de Richard Castle (Castle, ABC, 2009-) sont publiés par Hyperion pour la version anglaise et par City pour la traduction française; des bandes dessinées racontent le passé de William Bell et de Walter Bishop, les savants fous de Fringe (Fox, 2008-2013) ; Ma sorcière bien aimée (Bewitched, ABC, 1964-1972) a été ressuscitée au cinéma; des dizaines de romans continuent les aventures de Star Trek (NBC, 1966-1969)...

Nous appelons « constellations » de tels ensembles d'éléments, qui semblent participer d'une même histoire. Cette notion parait proche de celles de «transmédia » et de «monde fictionnel ». Dans un premier temps, nous explorons les caractéristiques des ensembles narratifs étudiés, des transmédias et des mondes fictionnels afin de vérifier s'il s'agit des mêmes phénomènes. L'examen montre que les

${ }^{1}$ Les webisodes sont des épisodes, la plupart du temps courts, diffusés sur Internet soit en parallèle d'une diffusion télévisuelle, soit entre deux saisons. 
constellations étudiées ne répondent pas encore aux exigences des récits transmédiatiques. Le concept de modèle satellitaire développé par Richard Saint-Gelais s'applique mieux à ces adaptations. Nous énonçons ensuite les propriétés de ces récits. Ces ensembles étant constitués d'éléments disparates, l'on peut se demander comment les lecteurs leur donnent cohérence. L'immersion fictionnelle décrite par Jean-Marie Schaeffer et l'expérience liée à la figure examinée par Bertrand Gervais sont utiles pour comprendre les processus en jeu. Enfin, une partie empirique basée sur des entretiens semi-directifs avec des amateurs de séries télévisées permet d'approcher leurs pratiques concrètes des constellations narratives. En effet, il nous semble important de dépasser les constats théoriques pour vérifier ce que les publics font réellement des récits qui se développent sur plusieurs supports. Des premières conclusions qui sont autant d'hypothèses de travail pour une recherche plus ambitieuse sur les usages clôturent cet article.

Deux précisions sont nécessaires avant d'entrer dans le propos. Nous ne nous intéressons qu'aux adaptations fictionnelles. Une multitude d'éléments prolongent le plaisir des séries : des objets, des sites d'analyse, des guides officiels et non officiels, des bandes annonces, etc. Ils ne seront pas pris en compte dans le cadre de cet article. De plus, nous n'étudierons que la partie officielle des constellations narratives, c'est-à-dire les éléments proposés et contrôlés par les auteurs et les producteurs des séries télévisées. Les prolongements développés par les fans ne seront donc pas abordés dans notre analyse. Pourtant, l'enquête menée montre que c'est peutêtre une erreur de notre part. En effet, les amateurs ne font pas nécessairement de distinction entre les éléments fictifs et les informations réelles. Ils ne semblent pas tous établir de hiérarchie entre ce qui est produit par les auteurs et ce qui émane des fans et que nous avons pourtant écarté dans un premier temps.

\section{Les mondes diégétiques transmédiatiques}

La plupart des auteurs qui s'intéressent aux récits transmédiatiques en relèvent trois caractéristiques importantes : la multiplication des supports, la création de mondes diégétiques, la participation des consommateurs. Selon Henry Jenkins,

[u]ne histoire transmédiatique se déploie à travers des plateformes médiatiques multiples, chaque nouveau texte apportant une contribution distincte et significative à l'ensemble² .

\footnotetext{
${ }^{2}$ Henry Jenkins, Convergence Culture. Where Old and New Media Collide, New York \& London, New York University Press, 2008, p. 97: «A transmedia story unfolds across multiple media platforms, with each new text making a distinctive and valuable contribution to the whole. » Traduction personnelle.
} 
Chaque entrée de la franchise est autonome (elle peut se consommer seule et il n'est pas nécessaire d'avoir vu ou lu un élément pour comprendre le suivant) et complémentaire ${ }^{3}$. C'est sur ce point que le transmédia se distingue du multimédia, du plurimédia ou du cross média ${ }^{4}$. En effet, chaque plateforme ne propose pas une déclinaison d'un même contenu qui serait traduit différemment en fonction des possibilités techniques et narratives du média. Au contraire, chaque texte $^{5}$ apporte quelque chose de neuf, "continue " l'histoire. "Consommer plusieurs médias permet une expérience plus profonde qui motive en retour à consommer encore ${ }^{6}$. » Henry Jenkins, analysant l'exemple de Matrix (réal. Andy et Lana Wachowski, 1999, 2003), souligne que les récits transmédiatiques sont prévus comme tels dès leur création.

Les auteurs du cahier Le Transmédia dans tous ses états édité par la Fondation Télécom constatent que le transmédia «se réduit dans certains cas à un simple lien entre deux contenus sur deux supports différents, sans vision globale, sans écriture nouvelle ", ce qu'ils considèrent comme une version affaiblie de ce type de récit. En effet, selon eux, " un des aspects fondamentaux du transmédia est bien le travail d'écriture de l'histoire ${ }^{7}$ ». Proposer des réalisations multiplateformes ne suffit donc pas à élaborer un transmédia, le projet narratif doit faire la différence. Selon Renira Gambaroto, les univers transmédiatiques sont " des constructions de mondes fictionnels ${ }^{8}$ », ce qu'Henry Jenkins nomme le " worldmaking ${ }^{9}$ ». L'auteur évoque une conversation avec un scénariste expérimenté qui lui apprend que, quand il a commencé, il fallait vendre une histoire car un bon film se créait sur la base d'une bonne histoire; puis, quand les sequels sont devenues à la mode, il a fallu vendre un personnage qui pouvait donner naissance à plusieurs films ; actuellement, il faut vendre un monde car un monde rassemble plusieurs personnages qui peuvent chacun porter des histoires ${ }^{10}$. David Peyron définit la création de monde comme :

\footnotetext{
3 Jenkins, p. 97-98.

4 Voir Fondation Télécom, "Transmédia, terme mouvant », in Le Transmédia dans tous ses états, Les Cahiers de veille de la fondation Télécom, mai 2012, p. 4 http://www.fondation-telecom.org/actualites/le-4e-cahier-de-veille-en-ligne-144, consulté le 10 décembre 2012.

5 Les mots «texte» et «lecteur» seront utilisés dans le sens large. Le mot «texte» désignera à la fois des productions textuelles, visuelles, audiovisuelles, multimédias. Le mot « lecteur » sera employé comme synonyme de spectateur, téléspectateur, internaute.

${ }^{6}$ Jenkins, p. 98 : « Reading across the media sustains a depth of experience that motivates more consumption. » Traduction personnelle.

7 Fondation Télécom, p. 4.

8 «Worldbuilding », Renira Gambarato, «How to Analyze Transmedia Narratives? », Conference New Media: Changing Media Landscapes, Saint Petersburg, 23 septembre 2012, http://prezi.com/fovzojrlfsno/how-to-analyze-transmedia-narratives, consulté le 9 décembre 2012.

9 Jenkins, p. 21 et 116-117.

${ }^{10}$ Jenkins, p. 116.
} 
le fait pour un auteur de construire, non pas une œuvre en tant qu'objet mais un monde total et indépendant du nôtre, un autre monde cohérent, qui serait ainsi d'une richesse telle qu'il serait développable à l'infini'11.

Anne Besson remarque que la fantasy et la science-fiction ont généré un nombre important de cycles de romans, ce qu'elle explique par « la volonté des auteurs et des lecteurs d'être confrontés à des mondes les plus complets et donc les plus immersifs possibles ${ }^{12} »$. Nous reviendrons sur la notion d'immersion, mais cette remarque d'Anne Besson nous emmène vers une troisième caractéristique généralement énoncée à propos du transmédia : la participation du lecteur.

Henry Jenkins considère que les récits transmédiatiques sont l'exemple le plus abouti de la convergence culturelle. Ce phénomène, selon lui, dépend avant tout de la volonté de participation des publics. Il oppose donc les anciennes pratiques culturelles aux nouvelles : les anciens consommateurs étaient passifs, prévisibles, isolés et silencieux, les nouveaux sont actifs, mobiles, connectés et bruyants ${ }^{13}$. Pour vivre pleinement l'expérience du monde fictif, les publics doivent devenir des chasseurs, rechercher chaque élément de l'histoire à travers différents médias, partager leurs découvertes avec les autres ${ }^{14}$. L'auteur développe longuement l'exemple de Matrix et souligne qu'il n'aurait pas pu découvrir la moitié des éléments qu'il présente sans la communauté des fans de cet univers ${ }^{15}$.

\section{Constellations, participation et simultanéité}

Dans un article précédent ${ }^{16}$, nous avons mis en lumière des caractéristiques qui nous semblent particulières aux constellations dont il est question dans cette contribution. Nous allons rapidement les résumer dans cette partie. Nous verrons qu'elles répondent en partie aux particularités des récits transmédiatiques, mais sans exploiter pleinement les potentialités narratives que ces derniers permettent. La série originelle reste prédominante, raison pour laquelle nous utilisons

${ }^{11}$ David Peyron, «Quand les œuvres deviennent des mondes. Une réflexion sur la culture de genre contemporaine à partir du concept de convergence culturelle », Réseaux, $\mathrm{n}^{\circ}{ }^{148}$ 149, 2008, p. 353 [p. 335-368], http://www.cairn.info/revue-reseaux-2008-2-p-335.htm, consulté le 10 décembre 2012.

${ }^{12}$ Anne Besson citée par Peyron, p. 352.

13 Jenkins, p. 19.

14 Jenkins, p. 21.

15 Jenkins, p. 132

${ }^{16}$ Sarah Sepulchre, «La constellation transmédiatique de Breaking Bad. Analyse de la complémentarité trouvée entre la télévision et Internet ", Essachess - Journal for Communication Studies, vol. 4 , $\mathrm{n}^{\circ} 7,2011$, p. 175-186, disponible en ligne :

http://www.essachess.com/index.php/jcs/article/view/111, consulté le 9 décembre 2012. 
le concept de «constellation» et non celui de transmédia pour caractériser ces ensembles.

Ces constellations sont développées sur différents médias. Les Experts (CSI : Crime Scene Investigation, CBS, 2000-) sont déclinés en série télévisée, en jeu de plateau, dans des romans. Glee (FOX, 2009-) est aussi une comédie musicale, des CD, des romans. Le site officiel de Breaking Bad permet à l'internaute de feuilleter l'album photo de la famille White, de regarder des webisodes ou de surfer sur les blogs de Hank ou Marie Schrader. Par ailleurs, les éléments qui composent l'ensemble appartiennent à des genres différents. Par exemple, la constellation Breaking Bad comprend des jeux, dont certains sont interactifs, ou des récits de vie (les blogs). Parfois les constellations mélangent même des éléments fictifs et non fictifs. Le site officiel de Sons of Anarchy (FX, 2008-) présente entre autres le blog d'un motard bien réel.

Une autre particularité des systèmes narratifs étudiés est la simultanéité. Dans Breaking Bad, Walter Jr. crée un site Internet pour récolter des fonds pour le traitement de son père atteint d'un cancer. $\mathrm{Au}$ moment même où le site est présenté dans la série, il est accessible pour les internautes. Durant les saisons d'Esprits criminels (Criminal Minds, CBS, 2005-), les téléspectateurs peuvent suivre Penelope Garcia sur Twitter. Quand Emily Prentiss disparaît lors d'un épisode, Penelope réagit en direct sur le réseau social. Parfois même, les personnages parlent en temps réel d'événements qui se déroulent dans la « vraie vie », à l'image de Patty Hewes (Damages), qui se plaint du nombre trop important de policiers en ville le jour où le président Obama visite New York (le 6 avril 2010).

Enfin, ces ensembles semblent encourager une participation des téléspectateurs, même si celle-ci reste minimale. Ils doivent se faire chasseurs, comme le signalait Jenkins (cf. supra), car ils doivent chercher et rassembler les différents éléments. Un exemple célèbre est le site internet de la compagnie aérienne Oceanic Airlines, mis en place par les producteurs de Lost (ABC, 2004-2010) et qui comprenait des indices permettant de décoder certains éléments de l'intrigue. Cette page n'était pas renseignée dans les épisodes de la fiction : ce sont les fans qui l'ont trouvée sur la toile. C'est aux lecteurs de faire la démarche de visiter le site de Breaking Bad afin de vérifier ce qu'il renferme, de suivre des profils Twitter ou d'acheter des novellisations. Cependant, au-delà de cette recherche, les concepteurs des constellations liées aux séries télévisées ne favorisent pas vraiment la participation des consommateurs. Au mieux, ceux-ci peuvent commenter des contenus, personnaliser certains programmes (par 
exemple, la vidéo interactive et personnalisable ${ }^{17}$ qui permet à l'internaute de vivre une scène de Breaking Bad de l'intérieur). Les fans ne peuvent, par exemple, pas contribuer à la constellation en proposant leurs propres productions. Tout ce qui relève des fanfictions n'est donc pas inclus dans ces constellations officielles.

Les constellations bâties autour des séries télévisées peuvent donc être considérées comme des récits transmédiatiques un peu frileux. Cette faible attention donnée à la participation des publics semble montrer que les créateurs désirent garder le contrôle sur leur fiction. Idéalement, les récits transmédiatiques ne devraient pas établir de hiérarchies entre les différents éléments qui les composent. Dans la vision de Matrix des frères Wachowski, les jeux vidéo sont aussi importants que les films ou les dessins animés. Les constellations que nous analysons semblent plutôt organisées sur le «modèle satellitaire » établi par Richard Saint-Gelais ${ }^{18}$. Dans cette schématisation, une fiction peut être prolongée par différents textes, mais le récit originel reste au centre du système. Chaque nouvel apport se réfère à cette fiction première. L'auteur remarque qu'étonnamment, jamais aucun prolongement narratif ne cite une autre suite ${ }^{19}$. Ceci établit de facto une hiérarchie d'autorité entre le récit originel et les textes qui le prolongent.

Un autre élément légitime ce premier récit, selon Richard SaintGelais: il s'agit de la différence d'autorité entre les auteurs des différents textes. L'analyste distingue le "canon», le "domaine officiel » et les « productions non autorisées ${ }^{20}$ ». Le canon est la zone la plus légitime et la plus contrôlée par les créateurs. Le domaine officiel rassemble les autres productions également régies par le copyright, mais bénéficiant d'une légitimité moindre. Richard Saint-Gelais cite l'exemple de la franchise Star Trek où seuls les films et les séries sont considérés comme canoniques par la Paramount alors que les autres productions (les novellisations, les dessins animés notamment) appartiennent au domaine officiel ${ }^{21}$. Le troisième ensemble, les productions non autorisées (parodies, fan-fictions, etc.), échappe au contrôle des auteurs. Ces éléments ne bénéficient d'aucune légitimité. Les conceptualisations de Richard Saint-Gelais semblent particulièrement bien décrire les constellations narratives construites autour des séries télévisées. Si les prolongements analysés sont légitimes et contrôlés par les créateurs (et appartiennent donc au

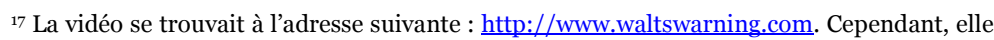
n'est plus disponible désormais. Il est possible d'en voir un enregistrement sur YouTube : http://www.youtube.com/watch?v=YWM5dLBTJZc.

${ }_{18}$ Richard Saint-Gelais, Fictions transfuges. La transfictionnalité et ses enjeux, Paris, Seuil, 2011, p. 313-322.

19 Saint-Gelais, p. 315 .

${ }^{20}$ Saint-Gelais, p. 373-434

${ }^{21}$ Saint-Gelais, p. 391-392. 
domaine officiel), il semble assez évident que la série originelle bénéficie d'une plus grande légitimité que n'importe quel autre élément. Les épisodes diffusés à la télévision constituent donc le centre du modèle satellitaire.

\section{L'expérience immersive des consommateurs}

Selon Henry Jenkins, la participation des consommateurs est une caractéristique essentielle de la culture développée sur des médias convergents. Anne Besson considère que les mondes narratifs répondent à une volonté d'immersion des lecteurs de science-fiction. À l'ère d'Internet, Frank Rose connecte directement participation et immersion :

Sous [l'influence d'Internet], un nouveau type de récit émerge - un récit raconté via plusieurs médias simultanément dans une narration non linéaire, un récit participatif, qui ressemble souvent à un jeu, un récit qui est conçu avant tout pour être immersif. C'est le « deep media» : des histoires qui ne sont pas simplement divertissantes, mais immersives, qui vous emmènent plus loin qu'un épisode de série d'une heure, un film de deux heures ou une publicité de 30 secondes le peuvent ${ }^{22}$.

\section{a) Immersion technique et fictionnelle}

Renée Bourassa a étudié les hyperfictions et les jeux narratifs. Ces objets ne sont pas tout à fait les nôtres. Cependant, parce que son corpus combine à la fois des aspects du jeu (qui nécessite des actions, une participation des joueurs) et des caractéristiques de la fiction (qui suppose une appréhension du monde et de l'intrigue), sa recherche est précieuse dans l'examen de cette notion d'immersion. Or l'auteure en détecte deux formes ${ }^{23}$.

L'immersion technique tente de plonger physiquement le lecteur/joueur dans le monde fictif en influençant son système de perception. Ces dispositifs tentent de faire disparaître le cadre de la fiction. Ce sont les cinémas Imax, les jeux qui se jouent avec des casques et des gants interactifs ou se déroulent dans des pièces recouvertes d'écrans. Dans ce cas, l'immersion passe par la simulation d'un monde et l'expérience que le participant fait de cet univers est

\footnotetext{
${ }^{22}$ Frank Rose, The Art of Immersion. How the Digital Generation is Remaking Hollywood, Madison Avenue, and the Way We Tell Stories, New York \& Londres, W. W. Norton, 2011, p. 3 : «Under its influence, a new type of narrative is emerging-one that's told through many media at once in a way that's nonlinear, that's participatory and often gamelike, and that's designed above all to be immersive. This is "deep media": stories that are not just entertaining, but immersive, taking you deeper than an hour-long TV drama or a two-hour movie or a 30-second spot will permit. » Traduction personnelle.

${ }^{23}$ Renée Bourassa, Les Fictions hyper-médiatiques. Mondes fictionnels et espaces ludiques, Montréal, Le Quartanier, 2010, p. 167-221.
} 
physique. Dans l'immersion technique, les actions du lecteur/joueur sont performatives. Il agit dans le cadre de la fiction, il est un actant de l'histoire. Ses actions sont diégétiques et il fait partie de l'intrigue.

L'immersion fictionnelle est mentale et fonctionne à partir d'associations d'idées. Selon Jean-Marie Schaeffer, quatre éléments caractérisent cet état d'immersion fictionnelle ${ }^{24}$. D'abord, dans cet état, le lecteur produit deux types d'activités : il perçoit et il imagine. La seconde est la plus importante. En effet, quand on parcourt un livre, notre attention est davantage concentrée sur l'histoire que sur le fait que l'on doit tourner des pages. Ensuite, durant l'immersion, deux mondes coexistent : le réel et le fictionnel. Le lecteur apporte dans sa lecture la totalité de ses représentations, de ses habitudes, de ses savoirs et il utilise ces éléments pour comprendre le récit. Il existe donc bien une véritable interaction entre ces deux mondes. L'immersion fictionnelle est, de surcroît, une activité homéostatique : elle se nourrit d'elle-même. Quand on lit ou quand on regarde un film, on prédit des événements, on formule des hypothèses, puis on repense le monde en fonction de ce qui se passe. Nous interprétons les récits et changeons constamment de compréhension. Enfin, l'immersion fictionnelle requiert un investissement émotionnel intense. Pour être réellement immergé dans un récit, nous devons nous sentir concernés par ce qui advient, par le destin des personnages. Jean-Marie Schaeffer réhabilite donc cet état d'immersion. Contrairement aux idées reçues, le lecteur ne s'oublie pas dans la lecture ou ne s'évade pas du réel grâce à la fiction, mais il établit des connections entre divers univers. L'auteur n'oppose d'ailleurs pas ce qui serait une lecture académique à une lecture fictionnelle (émotionnelle). Selon lui, pour lire un texte de manière critique, nous devons d'abord le comprendre. Or, pour comprendre un récit, nous devons d'abord le vivre, le ressentir, nous immerger. L’immersion fictionnelle est la première étape de la lecture savante.

Nous avançons l'hypothèse que les constellations jouent, comme les hyperfictions ou les jeux narratifs étudiés par Renée Bourassa, sur les deux types d'immersion. Les ensembles narratifs possèdent une dimension ludique, un aspect de "chasse au trésor ", car le lecteur doit trouver les éléments, ils ne sont pas donnés. On ne peut cependant pas affirmer que les constellations sont des simulations de monde car le cadre technique ne s'estompe pas et les actes posés ne sont pas vraiment des actions diégétiques. En revanche, on peut avancer l'idée que, dans ces systèmes, le cadre s'ouvre. La fiction entre plus profondément dans nos vies. Quand on peut recevoir un tweet d'un personnage, le voir apparaître dans nos amis Facebook, lire ses commentaires portant sur le monde réel, on peut parler d'une certaine forme de simulation même si elle n'est pas totalement immersive.

24 Jean-Marie Schaeffer, Pourquoi la fiction?, Paris, Le Seuil, 1999, p. 179-198. 
Pourtant, c'est vraisemblablement l'immersion fictionnelle qui est la plus investie par les constellations. Ces systèmes narratifs complexifient la perception des récits car il faut appréhender des textes plus épars et hétérogènes. Il faut également développer une activité imaginative plus intense pour créer des connections entre les éléments diégétiques. Les hypothèses de lecture énoncées sont également plus exigeantes car elles doivent convenir non seulement à l'échelle du micro-récit, mais aussi pour le monde narratif. Ces hypothèses supposent également de réactiver une mémoire du texte plus intense car tous les supports déjà consommés doivent être pris en compte. Jean-Marie Schaeffer postule que l'émotion est nécessaire afin de se sentir concerné par un récit. C'est probablement d'autant plus vrai pour la réception d'une constellation narrative. Enfin, il paraît évident que deux univers coexistent toujours dans la consommation des constellations, celui du réel et de la fiction. Ils s'imbriquent probablement de manière plus complexe puisque les différents textes apparaissent à des moments différents, de manière plus ou moins régulière, selon des modalités diverses. Le monde fictionnel accompagne ses lecteurs plus longtemps et, quand la constellation flirte avec le monde réel, s'invite dans leur quotidien.

\section{b) La lecture des constellations de la perception à} l'imagination

Il reste cependant une question à affronter. Les constellations produisent-elles des immersions différentes des textes unitaires? Peuton toujours parler d'immersion alors qu'on doit sortir du texte pour s'investir ensuite dans la lecture d'un second ? Le monde diégétique global ne pâtit-il pas de la fragmentation du récit? Le travail de Bertrand Gervais autour de la figure permet d'esquisser des pistes de réponse intéressantes.

Une figure, selon Bertrand Gervais, est un objet du texte - soit un objet au sens littéral du terme ${ }^{25}$ soit un personnage - que le lecteur perçoit, puis qui devient l'occasion d'une appropriation, d'une construction imaginaire. Ces deux moments (perception et imagination) sont essentiels dans la formation d'une figure. Bertrand Gervais la conçoit en trois temps : la figure-trace, car il doit y avoir un élément quelconque qui assure son existence; la figure-pensée, car l'objet doit engager le lecteur dans un processus symbolique ; enfin la figure-savoirs, car le lecteur l'interprète en fonction de ses

\footnotetext{
25 Il cite un cendrier dans un texte de Witold Gombrowicz intitulé Cosmos. Bertrand Gervais, Figures, lectures. Logique de limaginaire, Tome 1, Montréal, Le Quartanier, 2007, p. 15-16.
} 
connaissances encyclopédiques, de ses expériences personnelles et il l’intègre à son imaginaire ${ }^{26}$.

La figure est dynamique, non statique, elle génère de l'activité. C'est pourquoi Bertrand Gervais la considère comme une " expérience ${ }^{27}$ ». Le lecteur est essentiel à sa construction. Elle démarre de "presque rien ${ }^{28}$ » et c'est parce que le lecteur remarque l'objet, qu'il en garde une trace, qu'il se fait " museur ${ }^{29}$ » - autrement dit qu'il se perd dans ses pensées, les possibilités, les jeux d'associations -, qu'il en fixe ensuite les interprétations, que la figure existe. En ce sens, elle est un « objet auratique ${ }^{30}$ » c'est-à-dire un objet qui

déploie, au-delà de sa propre visibilité, ce que nous devons nommer ses images, ses images en constellations ou en nuages, qui s’imposent à nous comme autant de figures associées, surgissant, s'approchant et s'éloignant pour en poétiser, en ouvrager, en ouvrir l'aspect autant que la signification, pour en faire une œuvre de l'inconscient ${ }^{31}$.

Si on ne peut pas complètement assimiler la constellation à la figure, le processus de formation de cette dernière peut s'appliquer aux ensembles narratifs que nous étudions. Il semble que la série originelle peut être considérée comme l'objet-trace qui initie la figure et que les systèmes narratifs ménagent autant de chemins de déambulation possibles pour le téléspectateur-museur. Pour que l'ensemble s'actualise, il faut effectivement qu'un lecteur s'empare du texte premier, qu'il décide de s'y attarder, de faire des connexions, de penser, d'imaginer pour ensuite l'interpréter à la lumière de ses pérégrinations, de son encyclopédie personnelle, de son expérience. Dans une grande mesure, les constellations narratives n'ont de sens, de cohérence et même d'existence que si les téléspectateurs font ce travail d'imagination qui relie les différents textes.

\section{Que font les consommateurs des constellations narratives?}

Les éléments développés ci-dessus restent théoriques. Or cet article s'est donné pour mission d'explorer les pratiques des consommateurs. Dans cette dernière partie, nous allons synthétiser les résultats d'une étude exploratoire. Cette partie empirique demeure largement insuffisante et devra être prolongée par une étude plus

\footnotetext{
${ }^{26}$ Gervais, p. 32.

${ }^{27}$ Gervais, p. 38.

${ }^{28}$ Gervais, p. 17

29 Gervais, p. 47-48.

30 Bertrand Gervais reprend ici la notion d' «aura » énoncée par Walter Benjamin dans Charles Baudelaire (Paris, Payot, 1979) et relue par George Didi-Hubermann dans Ce que nous voyons, ce qui nous regarde (Paris, Minuit, 1991).

${ }^{31}$ Gervais, p. 75-76.
} 
rigoureuse. En effet, l'échantillonnage n'est pas représentatif et est trop restreint. Seuls des étudiants et une jeune enseignante ont été interrogés, uniquement des amateurs de séries qui consomment des prolongements sur d'autres supports. Ce sont donc des premières pistes de travail que nous allons énoncer.

Les personnes ${ }^{32}$ rencontrées (entre le 30 octobre 2012 et le 13 novembre 2012) sont dix étudiants inscrits en Sciences de l'Information et de la Communication à l'Université catholique de Louvain en Belgique 33 et une jeune enseignante, neuf filles et deux garçons. Tous regardent très régulièrement des séries télévisées. Parmi eux, neuf se déclarent amateurs de séries («mais pas fanatique ») et deux sont des fans («accro »). Tous sont devenus amateurs durant leur adolescence ou au début des études supérieures. Dix d'entre eux téléchargent ou regardent en streaming les séries sur Internet, en version originale et, pour la plupart, suivent la diffusion américaine (certains utilisent même des tableaux Excel ou des calendriers en ligne pour ne pas manquer d'épisodes). La majorité d'entre eux suivent entre 10 et 15 séries, ils regardent donc entre 1 et 3 épisodes par jour. Cependant, trois d'entre eux se limitent à une ou deux séries en même temps. Enfin, ils regardent les séries en solitaire ou, parfois, avec une personne «privilégiée » (copain ou copine, ami ou amie qui aime aussi les séries, frères ou sœurs). Pratiquement tous ressentent le besoin de parler de cette passion avec d'autres personnes 34 .

\section{a) Prolongements informationnels et fictionnels se confondent}

Nous souhaitions rencontrer des personnes qui consomment les séries et leurs prolongements fictionnels officiels, c'est-à-dire des textes qui étendent le monde et les intrigues et qui émanent des producteurs des séries. Cependant, bien que l'objet de la recherche semble avoir été clairement énoncé35, nous avons reçu des réponses de personnes qui ne regardaient que les séries. En fait, ces étudiants fréquentent des sites Internet spécialisés dans l'analyse des séries, certains sont abonnés à des profils Twitter d'acteurs ou achètent des magazines. Nous avons écarté ces personnes qui ne semblaient pas

${ }^{2}$ Dans un souci d'anonymat, elles seront uniquement désignées par leur prénom.

33 Ils sont soit en troisième année de baccalauréat, le premier cycle en Belgique (l'équivalent des licences en France), soit en première année de master, le second cycle (également nommé master en France).

34 Le profil et les pratiques de nos amateurs rejoignent sensiblement les usages des fans interrogés par Hervé Glevarec pour son livre La Sériphilie. Sociologie d'un attachement culturel, Ellipses, Culture Pop, Paris, 2012 ou par Clément Combes dans la communication "La sériphilie comme pratique partagée : la consommation de séries à l'heure d'Internet ", La série revisitée par le web, Journée d'étude du réseau S.E.R.I.E.S, Paris, 20 janvier 2012. 35 Nous avons lancé un appel à volontaire par e-mail via les listes d'étudiants de l’École de communication de l'Université catholique de Louvain. 
correspondre à la recherche. Cependant, malgré cette première sélection, deux étudiants qui ne consomment pas de prolongements se sont glissés dans le corpus. Célestine ne consomme pas de prolongement fictionnel. Elle considère que, si la novellisation n'apporte rien par rapport à la série, elle n'a pas d'intérêt à ses yeux ; mais que, si elle ajoute des éléments, elle ne comprend pas pourquoi ceux-ci ne sont pas dans la fiction première. Elle se sentirait, dans ce cas, trahie par la série. Manon n'aime pas lire et n'a pas le temps de chercher d'autres textes, elle ne revoit d'ailleurs jamais les épisodes une deuxième fois. Elle ne semble donc pas ressentir le besoin de vivre et revivre l'expérience fictionnelle.

$\mathrm{Si}$, dans un premier temps, nous avons tenté d'éviter de rencontrer les téléspectateurs ne consommant «que » des supports informationnels pour nous concentrer sur ceux qui parcourent les constellations narratives, il nous paraît, au terme de ces entretiens exploratoires, cependant moins évident d'établir une distinction forte entre ces deux types de prolongements. En effet, toutes les personnes interrogées (qu'elles consomment ou pas d'autres supports) ont évoqué les sites informationnels. Pour certaines d'entre elles, ces éléments participent à leur immersion fictionnelle. Célestine, par exemple, recherche les spoilers car elle aime avoir des attentes vis-à-vis de la fiction et vérifier comment les événements annoncés se produisent. Elle s'intéresse également aux acteurs qui incarnent les personnages. Les confidences qu'ils font sur leur manière d'interpréter un rôle influencent sa vision du personnage. Les éléments connus sur l'acteur apportent aussi de l'épaisseur au protagoniste. Par exemple, elle n'aime pas le profil Facebook de l'actrice Kate Walsh, qui incarne le docteur Addison Montgomery dans Grey's Anatomy (ABC, 2005-) et dans son spin-off, Private Practice (ABC, 2007-2013). L'actrice est un peu "fleur bleue » et ces éléments parasitent la réception du personnage, qu'elle perçoit comme plus cynique. Elle l'apprécie donc moins. Guillaume, quant à lui, vérifie des éléments liés aux lieux ou aux personnages inspirés de personnes réelles. Suite à la diffusion de Breaking Bad, il s'est promené, sur Google Earth, dans Albuquerque, la ville où se déroule l'intrigue. Il a vérifié les éléments biographiques et historiques liés à la série Deadwood (HBO, 2004-2006). Dans le même ordre d'idées, Ioana s'est renseignée sur la lecture des expressions du visage et du langage non verbal après avoir regardé Lie To $\mathrm{Me}$ (FOX, 2009-2011). La distinction entre domaine officiel et productions non autorisées semble également peu importante pour les téléspectateurs. Marine consomme beaucoup de fan-fictions quand elle ressent une frustration par rapport à la série. Elle recherche les textes en fonction des personnages qui l'intéressent et des situations ou relations qu'elle aimerait voir développées. Cette lecture lui permet de mieux connaître les personnages, de se mettre à leur place. Elle ressent 
moins ce besoin quand les univers initiaux sont déjà très complets, comme celui de Fringe (FOX, 2008-2013), par exemple.

Il apparaît donc que des éléments sur les acteurs, des univers alternatifs inventés par des fans, les villes réelles où se déroulent les actions peuvent également fournir des entrées dans ce que les lecteurs considèrent comme l'univers de la fiction. Finalement, la définition de ce qui appartient ou non à la diégèse est une donnée extrêmement personnelle.

\section{b) Les livres, descriptions plus détaillées}

Les supports les plus cités par les téléspectateurs sont les livres et les bandes dessinées. L'une a lu les novellisations de Plus belle la vie (France 3, 2004-)36 et le roman Lettre à Johanna supposément écrit par Blanche Marcy (l'un des personnages de la fiction)37. D'autres ont cité les livres liés aux séries True Blood (HBO, 2008-)38, Game of Thrones (HBO, 2011-)39, Gossip Girl (CW, 2007-2012)40, Vampire Diaries (CW, 2009-) ${ }^{41}$, Pretty Little Liars (ABC Family, 2010-) ${ }^{42}$ ou les bandes dessinées de Supernatural (The WB, The CW, 2005-)43, ou de The Walking Dead (AMC, 2010-)44. Que le livre soit le texte initial qui

${ }^{36}$ Plusieurs romans adaptent l'intrigue de la série télévisée. Une première série a été écrite par Claude Lambesc et publiée par les éditions Le Tigre bleu dans la collection Plus Belle La Vie. Des romans ont également été publiés par les éditions Fleuve Noir et Pocket Jeunesse. D’autres séries racontent le passé des personnages ou le Mistral des années 1970.

37 Blanche Marci, Lettre à Johanna, Les Éditions Comédia et Le Tigre Bleu, Nîmes et Paris, 2011.

${ }^{8}$ Charlaine Harris, La Communauté du Sud, Paris, J'ai Lu, 2001-2014. Il s'agit d'un cycle de treize romans publiés initialement sous le titre The Southern Vampire Mysteries chez Ace Books entre 2001 et 2013.

39 George R. R. Martin, Le Trône de fer, Paris, Pygmalion, 1998-. Il s'agit d'un cycle de douze romans (à l'heure actuelle), originalement publié sous le titre $A$ Song of Ice and Fire par Bartam Books depuis 1996.

${ }^{4}$ Cecily von Ziegesar, Gossip Girl, Paris, Fleuve Noir, 2006-2010. Il s'agit d'un cycle de seize romans initialement publiés sous le même titre par Little, Brown and Company entre 2002 et 2011.

${ }^{41}$ Lisa Jane Smith (puis Aubrey Clark), Journal d'un vampire, Paris, J'ai Lu, 2000 ; puis Hachette Jeunesse, 2009-2013. Il s'agit d'un cycle de dix romans publiés initialement sous le titre The Vampire Diaries entre 1991 et 2013 par Harper Paperbacks, puis 47North. ${ }^{42}$ Sarah Shepard, Les menteuses, Paris, Fleuve Noir, 2008-. Il s'agit d'un cycle de quatorze romans (à l'heure actuelle) publiés initialement sous le titre Pretty Little Liars par Harper Teen depuis 2006.

43 Il existe trois cycles de 6 tomes de bandes dessinées (comics) adaptés de la série : Peter Johnson (avec Geoff Johns pour le premier tome), Supernatural: Origins, La Jolla (California), Wildstorm, 2007 ; Peter Johnson et Rebecca Dessertine, Supernatural: Rising Son, La Jolla (California), Wildstorm, 2008 ; Andrew Dabb, Daniel Loflin, Supernatural: Beginning's End, La Jolla (California), Wildstorm, 2010.

44 Robert Kirkman et Tony Moore, The Walking Dead, Berkeley, Image Comics, 2003-. Il s'agit d'une série de bandes dessinées comptant actuellement 108 numéros publiés en 18 volumes sous le titre général The Walking Dead. La série a été traduite par Edmond Tourriol et publiée par Semic puis par Delcourt pour la version française depuis 2007. 
a ensuite suscité une adaptation en série ou une novellisation qui reprend l'univers d'un feuilleton ne semble pas être une donnée importante. Cependant, si des éléments divergent entre les deux textes, certains s'en tiennent à la version de l'auteur initial (par exemple, les livres de George R. R. Martin plutôt que la série télévisée Game of Thrones).

Dans tous les cas, séries et romans sont liés. Par exemple, les livres pallient le manque d'épisodes. Les informations délivrées par le roman réduisent la frustration ressentie quand la série s'arrête entre deux saisons. Julie déclare n'avoir aucune patience et lit les livres si elle n'a pas les prochains épisodes pour savoir ce qui se passe. Lora parle de « récit d'attente » qu'elle consomme en attendant la saison suivante.

Souvent le livre permet de plonger plus intensément dans le monde. La plupart apprécie le fait que le roman apporte des éléments supplémentaires. Guillaume et Ioana trouvent dans le livre A Game of Thrones des descriptions plus détaillées que dans la série. En revanche, Ioana a également lu le livre Gossip Girl, qu'elle n'a pas aimé car il était trop simple, trop direct, sans descriptions et présentant juste des dialogues. Julie trouve que les personnages, notamment les personnages secondaires, sont mieux mis en valeur dans les romans. Selon Céline et Marine, les livres permettent également d'accéder aux pensées personnelles des personnages.

Si le livre doit avoir une valeur ajoutée, il faut cependant qu'il soit conforme à l'univers. Céline n'a pas aimé Lettre à Johanna car le contrat de lecture était trop différent. Le livre était présenté comme relatant le réel et elle n'a pas adhéré à cette sortie de la fiction. Ioana a ressenti un problème similaire avec le livre Gossip Girl. Les personnages sont trop discordants et cela l'a empêchée de véritablement apprécier le livre. Julie, également, se méfie quand les histoires sont trop divergentes. Elle lit les romans Vampires Diaries, mais comme les personnages agissent différemment et que les relations amoureuses ne sont pas identiques, elle les parcourt après avoir vu les saisons de la série et sans mélanger les deux réceptions car elle ne veut pas gâcher son plaisir.

Le livre permet aussi une autre relation à l'univers. Florence considère qu'elle a plus de temps pour imaginer les actions des personnages car les décors sont déjà plantés par la série. Julie déclare ressentir les choses différemment quand elle lit, elle est moins passive. On peut consommer le roman à son rythme, sans être contraint par la diffusion télévisuelle (Céline) ; on peut également lire dans différents endroits comme le train ou durant les pauses au travail (Lora).

D'autres supports fictionnels sont cités par les amateurs mais ils s'y attardent moins. Florence et Guillaume évoquent les DVD. Florence achète certains coffrets de séries qu'elle veut revoir mais dont les épisodes ne sont plus disponibles en streaming. Guillaume a une 
consommation très précise des DVD Blue Ray de Game of Thrones, qui lui permettent d'ausculter finement l'image et d'apprécier les détails des armes, des costumes, des décors. Ioana a vu les films de Sex and the City (HBO, 1998-2004) lors d'une soirée entre filles, mais elle ne les a pas aimés du tout. Ils étaient amusants, mais n'apportaient rien d'intéressant car deux heures, c'est, selon elle, trop court pour vraiment exploiter les personnages. Julie, Lora et Marine citent des prolongements sur Internet comme les blogs de Dawson (The WB, 1998-2003), les webisodes de Cougar Town, le site de Gossip Girl ou le fil Twitter de Brittany de Glee. Ces éléments sont de nouveau peu commentés, soit parce qu'ils ne sont finalement pas si intéressants que cela (le site de Gossip Girl), soit parce que c'est juste amusant (les réflexions de Brittany sur Twitter ou les webisodes de Cougar Town). Guillaume s'intéresse quant à lui aux cartes géographiques. Il attend avec impatience de pouvoir s'offrir un recueil de cartes du monde de Game of Thrones. Il aime avoir des repères et trouve que ce monde particulier est décrit de manière très géographique dans le livre et la série ; il se réjouit de s'approprier la cartographie des lieux.

\section{c) Quand le monde narratif fait partie de la vie réelle}

L'une d'entre eux s'approprie de manière très personnelle l'univers fictionnel. Elle est très sensible aux musiques, aux vêtements et à certaines petites phrases. Dès que l'épisode se termine, elle cherche les groupes entendus sur Internet. Contrairement à d'autres (Célestine ou Céline), qui ajoutent "simplement » ces chansons à leurs playlists personnelles, Emmanuelle a développé une réelle expertise en s'intéressant aux chanteurs moins connus. Selon elle, chaque série possède une empreinte musicale particulière et elle peut les reconnaitre. Une bande musicale bâclée peut véritablement diminuer le plaisir qu'elle ressent en regardant les épisodes.

Elle aime également repérer les vêtements de certains personnages et les rechercher ensuite. Elle ne copie jamais l'intégralité d'un style, mais s'approprie des accessoires, comme un serre-tête par exemple. Personne, sauf elle, ne peut faire le lien entre ce détail vestimentaire et la série ou le personnage. C'est donc un plaisir totalement personnel.

Enfin, elle est souvent marquée par des petites phrases entendues. Il peut s'agir de citations en voix off (comme celles qui ouvrent et clôturent Grey's Anatomy ou Esprits criminels), mais aussi de parties de dialogues. Elle les retient, les note, les enregistre dans son téléphone portable. Elle les relit souvent et se souvient des situations, des personnages. Cela lui permet de réfléchir à sa vie. Certaines citations représentent un soutien dans des moments difficiles. En définitive, sa vie est teintée par une multitude de détails issus des séries qu'elle apprécie. La fiction imprègne son réel. 


\section{d) Ce que les constellations apportent}

La consommation de différents supports permet de prolonger le plaisir pour beaucoup d'entre eux. Florence déclare être parfois déçue, mais quand les éléments sont de bonne qualité, cela permet vraiment de redécouvrir une série. Elle adore les récits, les histoires et les mondes transmédiatiques et ressent une véritable jubilation quand elle explore les différents textes. Florentin aime s'immerger dans un univers, c'est pour cela qu'il ne regarde qu'une série à la fois. Les livres permettent de rendre cette immersion plus forte. Ioana et Julie aiment avoir plusieurs points de vue sur une histoire, explorer un monde à partir de différentes entrées. Guillaume trouve que les descriptions plus détaillées et l'accès à l'intériorité des personnages offrent d'autres manières d'entrer dans un univers et que lecture et vision se complètent.

Beaucoup d'amateurs considèrent que la consommation d'autres supports influence en retour la vision de la série. Emmanuelle est tellement sensible aux musiques que la qualité de la bande originale change sa vision de la série. Elle trouve que les histoires des Frères Scott (One Tree Hill, The WB, The CW, 2003-2012) sont peu intéressantes, mais elle regarde cette série parce que les chansons sont très bonnes. A l'inverse, si la musique ou les dialogues ne sont pas bons, cela dévalorise la fiction. Florentin et Ioana comprennent mieux les personnages après avoir lu les livres. Ioana déclare être plus « dans leur tête ».

Cela ne signifie pas que les amateurs considèrent nécessairement l'univers diégétique dans son ensemble. Pour Céline, Emmanuel, Ioana, Julie, le support principal reste la série. Les autres textes viennent en complément. Pour d'autres, cela dépend de la qualité des supports, de l'écriture, de l'approfondissement apporté. Dans les meilleurs cas, il n'y a pas de hiérarchie d'importance entre les éléments narratifs. Pour Marine, cela dépend aussi des séries. Elle n'apprécie pas du tout la série Glee car les scénaristes exploitent, selon elle, très mal les personnages. Alors, dans ce cas, les fan-fictions sont plus importantes et elle suit la série uniquement pour garder le fil. En revanche, elle considère que l'univers de Game of Thrones est véritablement construit par la série et les livres. Florentin, Guillaume et Lora considèrent également que tous les supports sont importants s'ils sont de bonne qualité.

Enfin, si les amateurs aiment parler de leur série préférée, la relation avec les autres supports est plus intime et moins partagée. Ceux qui en discutent avec d'autres ne le font qu'avec des 
interlocuteurs privilégiés (la personne qui regarde la série avec eux, les autres membres de la fandom, l'ami qui a offert le livre, un interlocuteur dont on se rend compte qu'il s'y connaît aussi). Mais d'autres ne parlent pas de cette consommation additionnelle. Céline considère que c'est un plaisir coupable et qu'on la prendrait pour une folle si on savait qu'elle lit les novellisations de Plus belle la vie. Florence énonce un constat très proche. Selon elle, c'est moins dangereux de parler des séries que des autres supports. Elle ressent également une certaine gêne à parler des livres de True Blood car ils sont parfois érotiques et elle ne souhaite pas partager cet aspect. Emmanuelle a tissé un rapport tellement personnel avec certains éléments des séries qu'elle les protège jalousement. Une de ses sœurs à qui elle reprochait d'avoir téléchargé une chanson issue d'une série lui a fait remarquer que les musiques ne lui appartenaient pas. C'est assez révélateur de l'appropriation forte qu'elle développe. Les gens qui la connaissent bien savent qu'elle a développé une certaine expertise concernant les musiques, mais personne ne sait pour les vêtements et les petites phrases.

\section{Conclusion}

$\mathrm{Au}$ terme de cette première recherche exploratoire, il est donc possible d'énoncer quelques résultats et de les confronter aux apports théoriques pour parfois les nuancer. C'était l'un des objectifs majeurs de cet article. Nous discutons principalement dans cette conclusion trois points qui nous semblent particulièrement marquants : l'importance du monde fictionnel, l'érosion de la frontière entre réel et fiction, l'appropriation spectaculaire de l'univers qui est effectuée par les lecteurs.

La rencontre avec les amateurs de constellations narratives confirme que c'est bien un plaisir de la diégèse, une envie de se plonger dans l'univers ou de ne pas le quitter qui motive leurs pratiques. Les épisodes, les livres, les musiques, les DVD, les prolongements sur Internet sont perçus comme des éléments d'un même monde. Les constellations sérielles fonctionnent donc bien sur un principe immersif identique à celui que décrit Anne Besson pour les amateurs de science-fiction ( $c f$. point 1). D'ailleurs, les différents textes doivent être dans un style identique et utiliser des personnages proches afin que les lecteurs ne ressentent pas de rupture du contrat. Chacun de ces textes devient alors une nouvelle manière d'explorer l'univers. Par exemple, les livres permettent une approche plus descriptive ou une focalisation sur l'intériorité des personnages. La plupart des pratiques décrites par les personnes interrogées relèvent donc principalement de l'immersion mentale décrite par Renée Bourassa et Jean-Marie Schaeffer ( $c f$. point 3). Seule Marine reçoit régulièrement des tweets 
d'un personnage sur son ordinateur, ce qui pourrait être assimilé à une forme d'immersion technique.

Cependant, il n'est pas toujours évident de comprendre dans quel sens se produit la synthèse. Est-ce le lecteur qui ne veut pas sortir de la fiction ou est-ce la série qui fait irruption dans le quotidien, du moins dans des lectures très personnelles comme celle d'Emmanuelle ? Nous avons vu que n'importe quel élément peut être investi par les consommateurs comme un élément fictionnel, même des biographies, des plans Google ou des informations concernant les acteurs qui incarnent les personnages. Comme le pressent Jean-Marie Schaeffer, le réel et le fictionnel coexistent donc, dans des formes d'appropriation qui se révèlent beaucoup plus complexes que prévu. Chaque lecteur semble avoir la possibilité d'importer n'importe quel élément dans l'univers diégétique qu'il reconstruit. Il semble donc un peu abusif de décider par principe d'écarter des téléspectateurs qui ne consomment pas ce que nous classons dans le fictionnel. Dans des étapes ultérieures de la recherche, il conviendra d'intégrer dans le corpus les consommateurs de prolongements informationnels et de vérifier comment ils utilisent ces éléments.

Un autre élément apporté par la rencontre avec les amateurs de séries contredit l'un des constats établis durant la partie théorique de cet article. Nous affirmions alors que les constellations n'étaient pas tout à fait des récits transmédiatiques car la participation des lecteurs restait faible. Il apparaît désormais que nous devons nuancer ce propos. Les téléspectateurs peuvent effectivement peu contribuer au texte lui-même car les producteurs maintiennent une distinction forte entre le domaine officiel émanant d'auteurs autorisés et les productions d'autres personnes. Néanmoins, les fans s'approprient quand même l'univers. À partir des éléments ténus évoqués dans le paragraphe précédent - ce que Bertrand Gervais appellerait des figures-traces ( $c f$. point 3.b) - ils développent bel et bien une activité interprétative de l'ensemble du monde diégétique. Cette activité élaborée à travers un parcours de déambulations imaginaires et de jeux d'associations peut intégrer, nous venons de le rappeler, des éléments très disparates, dont des informations factuelles. Ce processus est peutêtre moins explicite que si les fans pouvaient déposer des nouvelles ou des vidéos sur les sites officiels des séries, mais il n'en reste pas moins une forme assez spectaculaire d'appropriation personnelle d'un univers. Ce phénomène est parfois tellement personnel et fort que certains éléments ne sont jamais rendus publics. Les amateurs parlent volontiers de la série avec autrui, mais discutent beaucoup moins leur consommation des livres, des $\mathrm{BD}$ ou des musiques. Si certains affirment que c'est parce qu'il s'agit d'un plaisir coupable, il semble évident que, pour d'autres, c'est la relation très intime nouée avec l'univers qui explique cette retenue. 
Il faut cependant constater que les constellations restent faibles sur un point: il demeure effectivement une hiérarchie entre les différents éléments. En effet, les prolongements n'ont de signification qu'en lien avec la série, suivant une structure proche du modèle satellitaire décrit par Richard Saint-Gelais ( $c f$. point 2). Les entrées de l'ensemble narratif ne sont donc pas aussi autonomes que les fragments composant les récits transmédiatiques décrits par Henry Jenkins. C'est particulièrement évident quand les lecteurs perçoivent des contradictions entre les divers constituants. C'est alors le texte originel qui fait le plus souvent loi. Il convient, ceci dit, de souligner que les lecteurs ne désignent pas toujours le même élément comme texte originel, notamment quand la série est adaptée d'un livre. Si le cas de Game of Thrones est assez clair car les fans savent que les livres sont antérieurs à la série, il n'en va pas nécessairement de même pour Vampire Diaries, par exemple. Quelques consommateurs, cependant, s'accommodent de ces contradictions car ils considèrent que tous les textes participent d'un monde plus global. Pour Marine même, la série peut être secondaire si les fan-fictions sont plus satisfaisantes. Bref, si le texte originel reste encore la référence, il n'est pas exclu que certaines appropriations contournent les distinctions entre canon, domaine officiel et productions non autorisées.

La rencontre avec les publics permet donc de porter un regard neuf sur les adaptations multimédiatiques des séries télévisées. La consommation d'univers diégétiques transmédiatiques semble être un processus complexe et très personnel qui peut remettre en question l'autorité des auteurs ou les distinctions entre réalité et fiction. $\mathrm{Au}$ moment de clôturer cet article, nous souhaitons cependant attirer une nouvelle fois l'attention du lecteur sur le fait que ces éléments devront être confortés par d'autres recherches. Il serait, par exemple, intéressant d'interroger des personnes qui n'aiment pas les séries, ainsi que des téléspectateurs qui ne consomment pas de prolongements ou uniquement des textes informationnels. Il faudrait également vérifier les consommations d'autres publics que les étudiants en Sciences de l'Information et de la Communication.

\section{Bibliographie}

BouRASSA Renée, Les fictions hyper-médiatiques. Mondes fictionnels et espaces ludiques, Montréal, Le Quartanier, 2010.

Combes Clément, "La sériphilie comme pratique partagée: la consommation de séries à l'heure s'internet », La série revisitée par le $w e b$, Journée d'étude du réseau S.E.R.I.E.S, Paris, 20 janvier 2012. 
Fondation TELeCom, Le Transmédia dans tous ses états, Les cahiers de veille de la fondation Télécom, mai 2012, http://www.fondationtelecom.org/actualites/le-4e-cahier-de-veille-en-ligne-144

GAMbarato Renira, "How to Analyze Transmedia Narratives? ", Conference New Media: Changing Media Landscapes, Saint Petersburg, 23 septembre 2012, http://prezi.com/fovzojrlfsno/howto-analyze-transmedia-narratives

GervaIs Bertrand, Figures, lectures. Logique de l'imaginaire, Tome 1, Montréal, Le Quartanier, 2007.

Glevarec Hervé, La Sériphilie. Sociologie d'un attachement culturel, Paris, Ellipses, 2012.

Jenkins Henry, Convergence Culture. Where Old and New Media Collide, New York \& London, New York University Press, 2008.

PEYron David, «Quand les œuvres deviennent des mondes. Une réflexion sur la culture de genre contemporaine à partir du concept de convergence culturelle », Réseaux, $\mathrm{n}^{\circ} 148-149$, 2008, p. 335-368, http://www.cairn.info/revue-reseaux-2008-2-p-33.h.htm

Rose Frank, The Art of Immersion. How the Digital Generation is Remaking Hollywood, Madison Avenue, and the Way We Tell Stories, New York \& Londres, W. W. Norton, 2011.

SAINT-GELAIS Richard, Fictions transfuges. La transfictionnalité et ses enjeux, Paris, Seuil, 2011.

Schaeffer Jean-Marie, Pourquoi la fiction?, Paris, Le Seuil, 1999.

SEPUlChRE Sarah, "La constellation transmédiatique de Breaking Bad. Analyse de la complémentarité trouvée entre la télévision et Internet ", Essachess - Journal for Communication Studies, vol. 4, $\mathrm{n}^{\circ} 7,2011$, p. 175-186,

http://www.essachess.com/index.php/jcs/article/view/111

\section{L'auteure}

Sarah Sepulchre est chargée de cours à l'École de Communication de l'Université catholique de Louvain (Belgique). Elle y enseigne les méthodes de recherche, l'analyse des médias et la culture médiatique. Ses recherches portent principalement sur la fiction télévisuelle, la construction des personnages télévisuels, les relations entre réel et fiction dans les séries, les études de genre 
appliquées aux séries et les pratiques scénaristiques. Elle participe aux travaux de l'Observatoire du récit médiatique et du réseau S.E.R.I.E.S. Elle est membre de Sophia (www.sophia.be) et vice-président de l'Association internationale des chercheurs en littératures populaires et cultures médiatiques (http://www.flsh.unilim.fr/lpcm). 